\title{
CARACTERIZACIÓN DEL EMPRENDIMIENTO EN BRASIL
}

\section{CHARACTERIZATION OF ENTREPRENEURSHIP IN BRAZIL}

\author{
Emigdio Larios Gómez*
}

*Doctor en Ciencias Administrativas. Benemérita Universidad Autónoma de Puebla. ORCID: https://orcid.org/0000-0002-3514-1319.

Dirección para recibir correspondencia: herr.larios@gmail.com 
CARACTERIZACIÓN DEL EMPRENDIMIENTO EN BRASIL.

\section{RESUMEN}

OBJETIVO: Describir las características del emprendimiento en Brasil, como ha evolucionado y su impacto actual en la economía y en los negocios y así identificar el perfil del emprendedor brasileño.

MATERIAL Y MÉTODO: Se trata de una investigación exploratoria basada en el análisis documental de contenido, se desarrolló con base en el paradigma cualitativo y consistió en buscar, seleccionar, analizar y sistematizar información secundaria de artículos científicos publicados en revistas indexadas y de reportes económicos publicados de organismos internacionales.

RESULTADOS: Se presentan los resultados en cuatro aspectos importantes: a) desencadenamiento del emprendimiento en Brasil, b) ranking mundial de emprendimiento, c) el Brasil emprendedor y d) perfil del emprendimiento en los brasileños.

CONCLUSIONES: Se puede concluir que el emprendimiento es tan importante en las Pequeñas y Medianas Empresas (PyMEs) y en los mercados locales, como también, en las grandes empresas y en los mercados nacionales e internacionales, igualmente debe ser considerada en este mismo nivel de importancia para las empresas públicas, las empresas sociales y las organizaciones privadas. Existe una mínima diferencia entre hombres y mujeres como agentes de emprendimiento en Brasil, con una edad de 25 a 34 años, donde los hombres son más activos que las mujeres, y aquellos que sólo poseen la enseñanza fundamental completa.

PALABRAS CLAVE: Emprendimiento. Brasil. Caracterización. Perfil emprendedor.

\section{ABSTRACT}

OBJECTIVE: To describe the characteristics of entrepreneurship in Brazil, the way it has evolved and its current impact on economy and on business; and also to identify the profile of Brazilian entrepreneurs. 
CARACTERIZACIÓN DEL EMPRENDIMIENTO EN BRASIL.

MATERIAL AND METHOD: This is an exploratory qualitative research based on Moustakas's phenomenological design and with the support of the content analysis technique, which consists of selecting, analyzing and summarizing secondary information in scientific articles from indexed magazines and economic reports from international organisms.

RESULTS: The findings are presented in four categories: a) unchaining of entrepreneurship in Brazil, b) world entrepreneurship ranking, c) The Brazilian entrepreneur and d) entrepreneurship profile of Brazilans.

CONCLUSIONS: It can be concluded that entrepreneurship is relevant in Small and Medium Enterprises [SME's] as well as in local markets. But also, in large enterprises as those in national and internationales markets, and it must be considered in this very level of relevance for public enterprises, social enterprises and private organizations. There is a minimum difference between men and women as entrepreneurship agents in Brazil, eventhough men are more active than women, aged between 25 to 34 years old alongside with a complete fundamental teaching.

KEY WORDS: Entrepreneurship. Brazil. Characterization. Entrepreneur's profile.

\section{Antecedentes del emprendimiento}

Según Hisrich \& Peters (2002), el emprendimiento se caracteriza por una capacidad de identificar oportunidades y crear algo innovador bajo las condiciones de incertidumbre, asumiendo los riesgos en ellos implicados. El proceso de emprendimiento tiene como resultado una nueva manera de realizar procesos de trabajo de un nuevo producto, ya sea un servicio, bien, idea o experiencia; ya que no sólo para emprender se requiere de crear nuevos productos tangibles, también en la actual economía terciaria (economía de intangibles), el factor de emprendimiento también incluye a las personas que investigan información o ideas sobre procesos de producción eficientes, así como nuevas formas organizativas.

Desde la perspectiva de Schumpeter (1961b), la actividad de emprendimiento implica innovación en la introducción de un nuevo producto, organización o proceso, lo que a su vez 
CARACTERIZACIÓN DEL EMPRENDIMIENTO EN BRASIL.

genera un proceso de destrucción de lo pasado a lo actual. Schumpeter (1961a) se refiere a destrucción al proceso de construcción creativa en el desarrollo de los nuevos productos, los nuevos métodos de producción y los nuevos mercados que sustituye lo viejo por lo nuevo, y como agente central de este proceso figura del empresario. El empresario no siempre puede ser emprendedor que crea innovaciones, y haciendo la diferenciación del emprendedor y del dueño de la empresa -a la que no siempre hay correspondencia- este es como una especie de jugador que juega por el placer de jugar y en que las ganancias materiales son como trofeos que demuestran su superioridad.

[...] llamamos emprendedores no sólo a aquellos hombres de "negocios" independientes, de una economía mercantil [...] como también todos los que, realmente llenan, esa función que definida; aunque, como está siendo la regla, sean empleados "dependientes" de una compañía [...] por otra parte, nuestro concepto es más restringido que el tradicional, por el hecho de no abarcar a todos los directores de firmas, gerentes o industriales que, simplemente, sólo dirigen un negocio establecido [...]. (Schumpeter, 1961, 103-104).

En este proceso de emprendimiento, han surgido estilos o tipos de emprendedores: 1) el emprendedor innovador, que crea nuevas industrias y, por esta razón, provoca cambios estructurales relevantes en la economía (Schumpeter, 1950); 2) el emprendedor comercial que se beneficia de las oportunidades de lucro (Kirzner, 1973); 3) el emprendedor que se aprovecha del elemento de incertidumbre (Foss \& Klein, 2017); y, 4) el emprendedor productivo y no productivo que sólo emprende por emprender (Baumol, 1990). En cualquiera de estos tipos o perfiles, puede decirse que el emprendedor tiene una visión; es decir, un espíritu empresarialemprendedor, capaz de actuar ante los emprendimientos de alto riesgo desde el descubrimiento de oportunidades empresariales y la explotación de estas mismas oportunidades (Gartner, 1988; Shane \& Venkataraman, 2000). Lo anterior, es porque el emprendedor-empresario, es: 1) responsable del crecimiento económico y el desarrollo social, 2) con la innovación dinamiza la economía, 3) emprender implica la idea de la sostenibilidad y tratar no sólo de individuos, sino también con comunidades, ciudades, regiones, y hasta países y, 4) es la mejor arma contra el desempleo (Dolabela, 1999). 
CARACTERIZACIÓN DEL EMPRENDIMIENTO EN BRASIL.

\section{MATERIAL Y MÉTODO}

Este trabajo nos acerca a variables económicas, sociales y actitudinales del emprendimiento, desde la perspectiva cualitativa como lo menciona Bonilla, Hurtado \& Jaramillo $(2009,37)$ "si los aspectos se relacionan con el ordenamiento social y las dimensiones objetivas, la estrategia más indicada es la cualitativa". Con base en el objetivo de la presente investigación se describen las características del emprendimiento en Brasil, como ha evolucionado y su impacto actual en la economía y en los negocios. Se trata de una investigación exploratoria basada en el análisis documental de contenido (G. Hernández, Jacobo, Anaya, y J. Hernández, 2015), y se desarrolló con base en el paradigma cualitativo (Bernal, 2006), y consistió en buscar, seleccionar, analizar y sistematizar información secundaria de artículos científicos publicados en revistas indexadas y de reportes económicos publicados de organismos internacionales. Asimismo, se ha desarrollado el método fenomenológico de Moustakas (1994). Es así que, la investigación se realizó en cuatro pasos:

1. Preparación de la recolección de datos.

- Formular la pregunta, definir los términos de la pregunta: ¿Cuál es la característica o perfil del emprendimiento en Brasil?

- Hacer una revisión de la literatura y determinar la naturaleza original del estudio.

2. Recolección de datos.

- En fuentes secundarias.

3. Organizar, analizar y sintetizar los datos.

- Consistió en desarrollar descripciones textuales y estructurales; componer descripciones textuales y estructurales; y realizar una síntesis de los significados y esencias textuales y estructurales

4. Resumen, implicaciones y resultados.

- Resumir todo el estudio y Relacionar los hallazgos del estudio y diferenciarlos de lo hallado en la revisión de la literatura.

\section{RESULTADOS}

Con base en el proceso de análisis de contenido a través del método fenomenológico, se presentan los resultados en cuatro aspectos importantes: a) desencadenamiento del emprendimiento en Brasil, b) ranking mundial de emprendimiento, c) el Brasil emprendedor, y d) perfil del emprendimiento en los brasileños. 
CARACTERIZACIÓN DEL EMPRENDIMIENTO EN BRASIL.

\section{Desencadenamiento del emprendimiento en Brasil}

El emprendimiento en Brasil tiene sus inicios en los años de 1990 con el surgimiento del Servicio Brasileño de apoyo a las Micro y Pequeñas Empresas (SEBRAE ${ }^{1}$ ) y la Sociedad Brasileña para Exportación de Software (SOFTEX²). Ambas son entidades que tiene como finalidad auxiliar y dar soporte al emprendedor en su actividad empresarial. Dornelas (2005), destaca que el SEBRAE es una entidad que propone incentivar la acción emprendedora brasileña y es un campo amplio de conocimiento para muchos ejecutivos, entre éstos los pequeños empresarios. Diez años después (1990-2000), una encuesta realizada por el Global Entrepreneurship Monitor (GEM $\left.{ }^{3}\right)$, presentaba a Brasil como referencia en el ramo de apertura de un nuevo negocio. Es así que desde el 2003, uno cada ocho adultos (que representaba el 13,2\% de la población); es decir, 14 millones de brasileños eran parte de las nuevas actividades comerciales (Dornelas, 2005).

\section{Ranking mundial de emprendimiento}

Recientemente, el Informe Condiciones Sistemáticas para el Emprendimiento Dinámico 2017 en América Latina (ICSEd-Prodem ${ }^{4}$ ) publicado por la Universidad Nacional de General Sarmiento en Argentina, describe a los países Latinoamericanos más emprendedores. Este estudio abarca una visión comparativa a nivel internacional de los tres países más emprendedores; en primer lugar, del ICSEd-Prodem (2017) está Singapur, con un índice del 67.93, seguido de Estados Unidos con 67 y Finlandia con 65.70. Por lo que respecta a Latinoamérica, Chile es el primer lugar en el continente y ubicado en el puesto 33 en el parámetro internacional, con un índice de 30.70, seguido de Brasil con el puesto 38 y un índice de 34.17, subiendo un peldaño en comparación al 2016 que ocupaba el $4^{\circ}$ lugar; y en tercer lugar Argentina, en el puesto 39 y un índice de 33.77. A estos países le siguen México (puesto 42), Costa Rica (puesto 43), Uruguay (puesto 44), Colombia (puesto 45), Perú (puesto 48), El Salvador (puesto 54), República Dominicana (puesto 55), Bolivia (puesto 56), Ecuador (puesto 57), Panamá (puesto 58), Venezuela (puesto 59) y Guatemala (puesto 60) (Kantis, Federico \& Ibarra, 2017).

\footnotetext{
${ }^{1}$ por sus siglas en portugués: SEBRAE -Serviço Brasileiro de Apoio às Micro e Pequenas Empresas.

${ }^{2}$ por sus siglas en portugués: SOFTEX -Associação para Promoção da Excelência do Software Brasileiro.

3 Por sus siglas en inglés: GEM -Global Entrepreneurship Monitor.

4 Índice de Condiciones Sistémicas para el Emprendimiento Dinámico de Prodem - ICSEd-Prodem. LARIOS-GÓMEZ E.
} 
CARACTERIZACIÓN DEL EMPRENDIMIENTO EN BRASIL.

Para el Global Entrepreneurship Index (GEI Index) del (GEDI ${ }^{5}$ ), en 2017 Brasil ocupaba la 98a colocación, detrás, por ejemplo, de Jamaica (93 $)$, Ghana (86), Irán (85a), Albania (80), Filipinas (76ª), Gabón (75a), Panamá (68ª), Líbano (63), Kazajstán (62a), Namibia (60ª), Costa Rica (58 $)$ y Botswana (52a). Pero en el informe del año anterior, Brasil se situaba en la posición 92 (también detrás de la mayoría de los países aquí citados) (GEDI, 2017). Actualmente, para 2018, el GEDI (2018), sigue ocupando el lugar 98 con 20.3 en el indicador. Además, dentro de los países latinoamericanos, Chile ocupa el primer lugar $\left(1^{\circ}\right)$ con 58.5 puntos, Puerto Rico en el segundo $\left(41^{\circ}\right)$ con 42.1 puntos y Colombia en el tercer lugar $\left(47^{\circ}\right)$ con 38.2 puntos.

Investigaciones ${ }^{6}$ recientes realizadas por Menezes, dos Santos y Tosi (2015), Queiroz y Vian (2016) y Moraes (2017) abordan la temática de estudio desde la perspectiva de la contribución del emprendimiento en el crecimiento económico de los Estados brasileños. Asimismo, estudios realizados por De Almeida, Sediyama y Santiago (2015), y Fochezatto y Céspedes (2015), hacen el abordaje desde un análisis regional en panel dinámico y econometría espacial. Al igual que otras instituciones de corte internacional, describen y cuantifican el emprendimiento en Brasil, destacando diferentes tasas de emprendimiento en relación con los asalariados, la influencia del desempleo como catalizador de emprendedores y las políticas públicas, en fomento al desarrollo económico como soporte del crecimiento de PyMEs para cada región brasileña.

Una investigación realizada por el GEM (2014), describe estadísticas entre 2004 y 2014, donde se observa que Brasil pasó del 23\% al 34,5\%, de los brasileños mayores de 18 años que tienen una empresa o están involucrados en la apertura de su propio negocio; es decir, incluye a todos los emprendedores nuevos y establecidos concentrados en la Tasa Total de Emprendimiento (TTE ${ }^{7}$ ); de este total, el $70 \%$ de los que buscaron un negocio propio, lo hicieron porque vieron oportunidades y no por necesidad (Okano \& Fernandes, 2017). Para el 2015, esta misma encuesta, el Global Entrepreneurship Monitor, describió que el 39\% de los brasileños mayores de 18 años tienen un negocio propio, lo cual ubicaba a los brasileños como los más emprendedores seguidos de China (26,7\%), Estados Unidos (20\%), Reino Unido (17\%), Japón $(10,5 \%)$, India (10,2\%), Sudáfrica $(9,6 \%)$, Rusia $(8,6 \%)$ y Francia $(8,1 \%)$ (GEM, 2015). Para el

\footnotetext{
${ }^{5}$ The Global Entrepreneurship and Development Institute (GEDI).

${ }^{6}$ Utilizando los datos de la Investigación Nacional por Muestra de Domicilio (PNAD) y modelos de elección discreta 2015-2016.

${ }^{7}$ Por sus siglas en portugués: Taxa de empreendedorismo total -TTE.

LARIOS-GÓMEZ E.

MAYO-AGOSTO 2019. Año 25, Número 72. Págs. 286-299 
CARACTERIZACIÓN DEL EMPRENDIMIENTO EN BRASIL.

2016, la TTE brasileños fue del 36,0\%, inferior comparada al año anterior (GEM, 2016) y para el 2017, sólo aumentó un 0.4\%, ubicándose en 36,4\% (ver figura 1) (GEM, 2017).

\section{El Brasil emprendedor}

En lo que se refiere a la tasa de emprendimiento, la región Sudeste tiene una TTE del 33,6\%; la región Sur 35,1\%; la región Norte 32,6\%; la región Nordeste 36,4\%; y, la región Centro-Oeste 33\% (de Almeida, Valadares \& Sediyama, 2017).

Con base en las informaciones obtenidas por el banco de datos de la National Household Sample Survey (PNAD) (IBGE, 2014), en la región Sudeste el 23\% de los individuos económicamente activos son emprendedores, donde los emprendedores se consideran tanto autónomos como los empleadores, en el Sur el 23\% son emprendedores, en el Norte 27\%, en el Nordeste $22 \%$ y en el Centro-Oeste el 23\%. Para Brasil, el 23\% de los individuos son empresarios (Dias, 2018).

En el 2015, la región Norte de Brasil se registró el mayor crecimiento con el 42\%, seguido por el Nordeste y Sur con el $28 \%$ y $22 \%$, respectivamente. La región Sudeste presentó un $50 \%$ de aperturas de empresas en el 2016, seguida por el Nordeste con el 19\%, y el Sur con $17 \%$ (Mota, Sobreira, Vale \& Nogueira, 2017).

Con 377 empresas, Fintech, en el 2017 Brasil se colocó como el país con el mayor ecosistema Fintech de América Latina, le siguen México y Colombia (Forbes, 2018).Asimismo, de acuerdo al indicador del Espiritu Emprendedor (Entrepreneurial Spirit Index), Brasil ocupa el lugar $11^{\circ}$ de los 54 países que integran la encuesta con un valor de 0.11 (GEM, 2018).

\section{Perfil del emprendimiento en los brasileños}

De los casi 50 millones de brasileños que emprenden y/o realizaron alguna acción de emprendimiento, 59.4\% emprendieron por oportunidad y el 39.9\% por necesidad, según el analista de Gestión Estratégica del SEBRAE (2017). El Instituto Brasileño de Calidad y Productividad $\left(\mathrm{IBQP}^{8}\right)$, reportó que en 2017 hubo un pequeño crecimiento en el número de emprendedores por oportunidad, situación contraria con los emprendedores por necesidad. En 2016 para cada emprendedor inicial por necesidad había 1,4 emprendedores por oportunidad y

\footnotetext{
${ }^{8}$ Por sus siglas en portugués: IBQP - Instituto Brasileiro de Qualidade e Produtividade. LARIOS-GÓMEZ E.
} 
CARACTERIZACIÓN DEL EMPRENDIMIENTO EN BRASIL.

para 2017 esa relación fue de 1,5; aunque es importante notar, que el emprendimiento por necesidad continúa incrementándose desde 2014, el cuál fue del 29\% (IBQP, 2017):

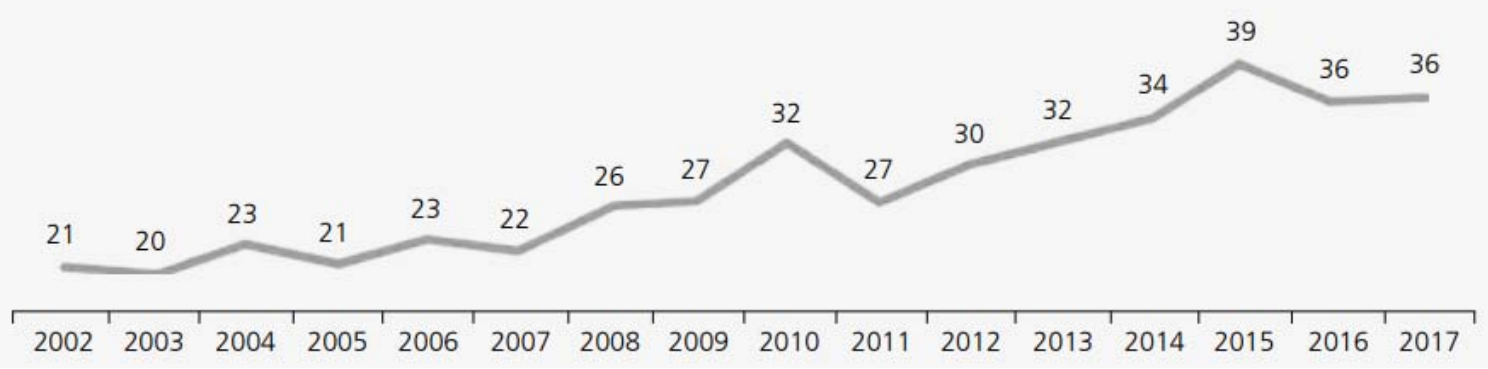

Figura 1. Tasa Total de Emprendimiento (TTE) en Brasil 2002-2017.

Fuente: Adaptada de GEM (2017).

- La Tasa de Emprendedores Iniciales (TEA ${ }^{9}$ ), compuesta por: emprendedores nacientes y nuevos, alcanzó el valor del 18\% en el 2014 (GEM, 2014), para el 2015 fue del 19\% (GEM, 2015) y se ha mantenido inferior en 17\% para 2016 y 2017 (GEM, 2017; Cacciotti, Hayton, Mitchell \& Giazitzoglu, 2016). Respecto a la Tasa de Emprendedores Establecidos (TEE $\left.{ }^{10}\right)$, en el 2014 fue de 17\%, en el 2015 llegó al 21\% y para 2016 y 2017 ha tenido el mismo comportamiento inferior que la TEA, siendo de $20 \%$ para ambos años (GEM, 2017). Por lo tanto, de cada 100 brasileños, aproximadamente 20 estaban involucrados con actividades emprendedoras (ver figura 2).

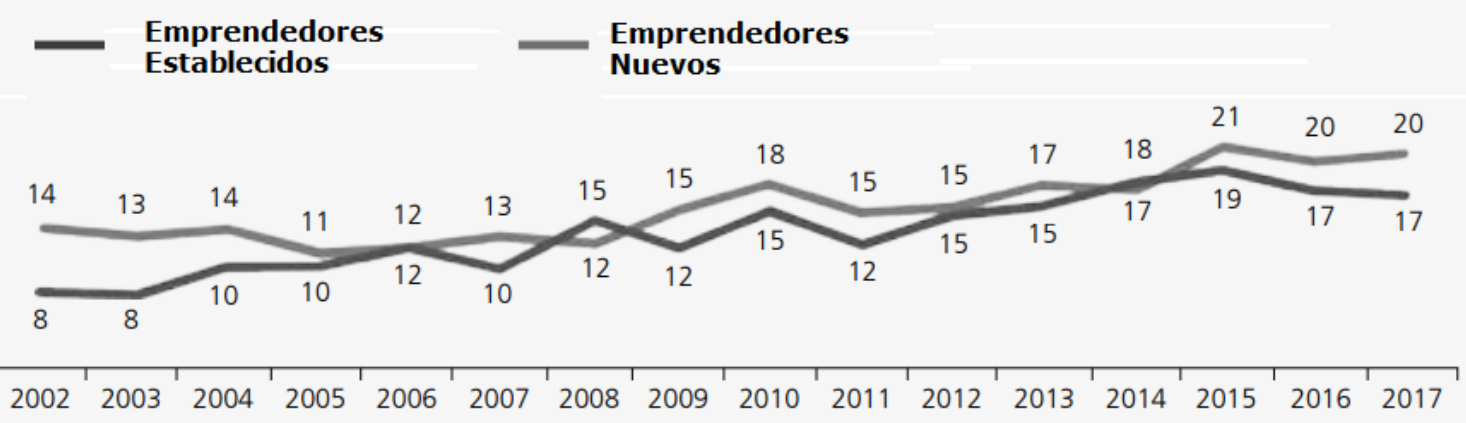

Figura 2. Tasa TEA y TEE en Brasil 2002-2017.

Fuente: Adaptada de GEM (2017).

\footnotetext{
${ }^{9}$ Por sus siglas en portugués: Taxa de empreendedorismo inicial -TEA (nascentes ou novos).

10 Por sus siglas en portugués: Taxa de empreendedorismo estabelecido -TEE.
} 
CARACTERIZACIÓN DEL EMPRENDIMIENTO EN BRASIL.

- El capital humano emprendedor en Brasil tuvo una evolución importante respecto a los países más desarrollados en Latinoamérica, aunque pierde posiciones respecto al 2012, producto de una caída que comienza en 2015, ya que cuesta avanzar y sostener los progresos en educación emprendedora a través de políticas públicas y 11 de los 15 países de la región, muestran un desmejoramiento en este sentido, como son: Panamá, Uruguay y Colombia. La disminución comenzó en 2015, luego de tres años de crecimiento que vivieron México, Perú, El Salvador y en menor medida Brasil, quien en 2017 tuvo un índice del 29.31 (ICSEd-Prodem, 2017).

- En Brasil, tener la perspectiva de una carrera profesional en empresa o en el servicio público, parece que está dejando de ser el sueño de los jóvenes brasileños, ya que cada vez se convierten en emprendedores desde su edad temprana. La encuesta GEM (2017) y del SEBRAE (2017), describe que creció del 50\% al 57\% los jóvenes de entre 18 y 34 años que abren una empresa (aproximadamente 15,7 millones).

- Caracterizando el perfil del emprendedor brasileño en 2017, se tiene que los jóvenes millennials de 18 a 24 años y de 25 a 34 años están creando nuevas empresas; es decir, son los nuevos emprendedores; y los brasileños de 35 a 54 años (generaciones X y Baby Boomers), ya son empresarios establecidos (los menos activos son de 55 a 64 años, (generación tradicionalista) (GEM, 2017). Del total de emprendedores en Brasil, el 37.9\% son hombres, donde un 19.9\% están iniciando (sólo 0.7\% más con respecto al 2016) y el 18.6\% están establecidos (1\% más que en 2016). Por lo que respecta al género femenino, el $35 \%$ son emprendedoras, donde el $20.7 \%$ son nuevas (aumentó el $0.8 \%$ con respecto al 2016) y el 14.4\% están establecidas (aumentó sólo el 0.1\% en comparación al 2016) (Assunção \& Anjos, 2018).

- Los emprendedores iniciales brasileños, se han concentrado en participar en sectores, como son los servicios orientados al consumidor (66.7\% en 2017 y el $69 \%$ en el 2016) y en el sector de la transformación (27\% en 2017 y el 24\% en el 2016), en estos dos mismos sectores, los emprendedores establecidos participan con sus actividades emprendedoras; es decir, en sectores como los servicios orientados al consumidor (48.7\% en 2017 y el $51.4 \%$ en el 2016) y en el sector de la transformación (42.1\% en 2017 y el $42 \%$ en el 2016) (SEBRAE-IBQP, 2017). 
CARACTERIZACIÓN DEL EMPRENDIMIENTO EN BRASIL.

\section{CONCLUSIONES}

El emprendimiento se hace más presente en las épocas de crisis económicas, como se puede comprobar en las estadísticas de Brasil; donde en algunos casos, los trabajadores se quedan desempleados y buscan una forma de sobrevivir a través de la apertura de un emprendimiento. Para el caso de Brasil, mientras que los indicadores económicos presentan índices negativos debido a la crisis económica, los indicadores de emprendimiento muestran una reacción inversa con índices positivos, caracterizando un aumento en este aspecto.

Por tanto, emprender implica para el emprendedor una serie de miedos, como la seguridad financiera, la capacidad personal, la capacidad para financiar la empresa, el potencial de la idea, el impacto social, la capacidad para ejecutar y el manejo eficiente de los costos.

El emprendimiento es importante en las Pequeñas y Medianas Empresas (PyMEs), así como en los mercados locales, pero también en las grandes empresas y en los mercados nacionales e internacionales, y debe ser considerado en este mismo nivel de importancia para las empresas públicas, las empresas sociales y las organizaciones privadas.

Existe una mínima diferencia entre hombres y mujeres como agentes de emprendimiento en Brasil, con una edad de 25 a 34 años, donde los hombres son más activos que las mujeres, y aquellos que sólo poseen la enseñanza fundamental completa.

\section{REFERENCIAS BIBLIOGRÁFICAS}

De Almeida, F. M., Sediyama, G. A. S. y Santiago, F. A. (2015). A Contribuição do empreendedorismo para o crescimento econômico dos Estados Brasileiros. In: Anais do XIII Encontra Nacional da Associação Brasileira de Estudos Regionais e Urbanos (ENABER), 2015, ABER, Curitiba-PR, 2015.

De Almeida, F., Lopes, J. y Santana, G. (agosto, 2017). A Contribuição do empreendedorismo para o crescimento econômico dos Estados Brasileiros. Revista de Empreendedorismo e Gestão de Pequenas Empresas, 6(3), 466-494. Recuperado de https://www.regepe.org.br/regepe/article/view/552/pdf 
CARACTERIZACIÓN DEL EMPRENDIMIENTO EN BRASIL.

De Assunção, J. y Delfino, M. (2018). Empreendedorismo feminino: Um estudo no estado de Minas Gerais. Revista GeTeC, 7(16). Recuperado de http://www.fucamp.edu.br/editora/index.php/getec/article/view/1369/954

De Oliviera, M., Do Carmo, M., Sampaio, M. y Da Costa (2017). Relações de influência de indicadores macroeconômicos na propensão ao risco de empreender. REGE-Revista de Gestão, 24(2), 159-169. https://doi.org/10.1016/j.rege.2017.03.006

Bernal, C. (2006). Metodología de la investigación. México: Pearson.

Cacciotti, G., Hayton, J., Mitchell, J. y Giazitzoglu, A. (mayo, 2016). A reconceptualization of fear of failure in entrepreneurship. Journal of Business Venturing, 31(3), 302-325. Recuperado de https://www.sciencedirect.com/science/article/abs/pii/S0883902616300027

Dias, G. (2018). Empreendedorismo e educação: o SEBRAE na escola. Revista Trabalho Necessário, 7(8).

Dolabela, F. (1999). Oficina do empreendedor. Río de Janeiro, Brasil: Sextante.

Dornelas, J. (2005). Empreendedorismo transformando ideias em negócios. Río de Janeiro, Brasil: Elsevier.

Forbes (junio, 2018). Brasil supera a México como el ecosistema más grande de fintech. Forbes. Recuperado de https://www.forbes.com.mx/brasil-supera-a-mexico-como-elecosistema-mas-grande-de-fintech/

Foss, N. y Klein, P. (marzo, 2017). Entrepreneurial discovery or creation? In search of the middle ground. Academy of Management Review, 42(4), 733-736. https://doi.org/10.5465/amr.2016.0046

Gartner, W. (1988). "Who is an entrepreneur?" is the wrong question. American journal of small business, 12(4), 11-32. https://doi.org/10.1177/104225878801200401

GEDI - Global Entrepreneurship and Development Institute. (2017). The GEI and Sub-Index Ranks of the First 25 Countries, 2017.

GEDI - Global Entrepreneurship and Development Institute. (2018). The GEI and Sub-Index Ranks of the First 25 Countries, Coord. Zoltán J. Ács László Szerb Ainsley Lloyd.

GEM - Global Entrepreneurship Monitor (2014). GEM 2014 Global Report - GEM Global Entrepreneurship Monitor. Brazil. Sponsoring Institution: Babson College, Babson Park, MA, United States Lead Sponsoring Institution and Founding Institution; Universidad del 
CARACTERIZACIÓN DEL EMPRENDIMIENTO EN BRASIL.

Desarrollo, Santiago, Chile; Universiti Tun Abdul Razak, Malaysia y Korea Entrepreneurship Foundation.

GEM - Global Entrepreneurship Monitor (2015). GEM 2015 Global Report - GEM Global Entrepreneurship Monitor. Brazil. Sponsoring Institution: Babson College, Babson Park, MA, United States Lead Sponsoring Institution and Founding Institution; Universidad del Desarrollo, Santiago, Chile; Universiti Tun Abdul Razak, Malaysia y Korea Entrepreneurship Foundation.

GEM - Global Entrepreneurship Monitor (2016). GEM 2016 Global Report - GEM Global Entrepreneurship Monitor. Brazil. Sponsoring Institution: Babson College, Babson Park, MA, United States Lead Sponsoring Institution and Founding Institution; Universidad del Desarrollo, Santiago, Chile; Universiti Tun Abdul Razak, Malaysia y Korea Entrepreneurship Foundation.

GEM - Global Entrepreneurship Monitor (2017). GEM 2017 Global Report - GEM Global Entrepreneurship Monitor. Brazil. Sponsoring Institution: Babson College, Babson Park, MA, United States Lead Sponsoring Institution and Founding Institution; Universidad del Desarrollo, Santiago, Chile; Universiti Tun Abdul Razak, Malaysia y Korea Entrepreneurship Foundation.

GEM - Global Entrepreneurship Monitor (2018). GEM 2018 Global Report - GEM Global Entrepreneurship Monitor. Brazil. Sponsoring Institution: Babson College, Babson Park, MA, United States Lead Sponsoring Institution and Founding Institution; Universidad del Desarrollo, Santiago, Chile; Universiti Tun Abdul Razak, Malaysia y Korea Entrepreneurship Foundation.

Salazar, G., Vargas, M., Hernandez, M. y Hernández, J. (2015). Estudio documental del Coaching en la industria del alojamiento mediante la cartografía conceptual. Revista Interamericana de Ambiente y Turismo-RIAT, 11(1), 37-54. http://dx.doi.org/10.4067/301 Hisrich R., Peters M. y Shepherd D. (2005) Entrepreneurship. Nueva York: McGraw-Hill. IBGDE - Instituto Brasileiro de Geografia e Estatística (2014). Estatísticas de Empreendedorismo - FTP - IBGE.

IBQP - Instituto Brasileiro da Qualidade e Produtividade. (2017). Relatório Executivo Empreendedorismo no Brasil.pdf - IBQP

Kantis, H., Federico, J. e Ibarra, S. (2017). Índice de Condiciones Sistémicas para el Emprendimiento Dinámico: Condiciones Sistémicas para el Emprendimiento Dinámico LARIOS-GÓMEZ E. 
CARACTERIZACIÓN DEL EMPRENDIMIENTO EN BRASIL.

2017 América Latina: avances y retrocesos en perspectiva [ICSEd-Prodem]. Recuperado de https://prodem.ungs.edu.ar/wp-content/uploads/2018/05/Resumen-2017.pdf

Kirzner, I. (1973). Competition and Entrepreneurship. Chicago, Estados Unidos: University of Chicago Press.

Menezes, G., dos Santos, V. y Tosi, F. (2015). Determinantes do empreendedorismo no Brasil: uma análise da escolha ocupacional e dos rendimentos. Revista Brasileira de Estudos Regionais e Urbanos,

Moraes, I. S. (2017). Diferencial de salário do empreendedor brasileiro. 2017. Dissertação (Mestrado em Economia Aplicada) - Universidade Federal do Rio Grande, Rio Grande, 2017.

Moustakas, C. (1994). Phenomenological research methods. London: Sage.

Okano, M. y Fernandes, M. (2017). A crise como oportunidade para o empreendedor Brasileiro. South American Development Society Journal, 3(7), 125-140. http://dx.doi.org/10.24325/issn.2446-5763.v3i7p125-140

Queiroz, V. y Antonio, G. (2016). Os Determinantes do empreendedorismo entre idosos brasileiros: evidências empíricas a partir dos dados das PNADs de 2003 e 2013. In: Anais do XIV Encontra Nacional da Associação Brasileira de Estudos Regionais e Urbanos (ENABER), 2016, ABER, Aracaju-SE, 2016. Recuperado de https://www.anpec.org.br/sul/2017/submissao/files_l/i2-

703c1be58b095ac5a31ae33c14984c12.pdf

Schumpeter, J. (1961b). Capitalismo, socialismo e democracia. Rio de Janeiro, Brasil: Fundo de Cultura.

Recuperado

de http://www.ie.ufrj.br/intranet/ie/userintranet/hpp/arquivos/100820171042_SchumpeterCap italismoSocialismoeDemocracia.pdf

Schumpeter, J. (1961a). Teoria do desenvolvimento econômico. Rio de Janeiro, Brasil: Fundo de Cultura.

SEBRAE - Serviço Brasileiro de Apoio às Micro e Pequenas Empresas (2017). Edital de Credenciamento SEBRAE/PR No 01/2017.

Shane, S. y Venkataraman, S. (2000). The promise of entrepreneurship as a field of research. Academy of management review, 25(1), 217-226. Recuperado de https://entrepreneurscommunicate.pbworks.com/f/Shane\%2520\%252B\%2520Venkat\%2 520-\%2520Ent\%2520as\%2520field.pdf 